\title{
Positive behaviour support for people with dementia
}

\author{
Alinka Fisher ${ }^{\mathrm{a}}$, Tim Connolly ${ }^{\mathrm{a}}$, Claire MC O’Connor ${ }^{\mathrm{b}, \mathrm{c}}$, Glenn Kelly ${ }^{\mathrm{d}}$
}

\author{
Affiliations: \\ ${ }^{a}$ Flinders University, College of Nursing and Health Sciences Adelaide, Australia \\ ${ }^{\mathrm{b}}$ HammondCare, Centre for Positive Ageing, Sydney, Australia, \\ ${ }^{c}$ The University of New South Wales, School of Population Health, Sydney, Australia \\ ${ }^{\mathrm{d}}$ Concept Psychology Services, Victoria, Australia
}

Declarations of interest: The authors (AF, TC, CMOC, GK) report no conflicts of interest to disclose. This research did not receive any specific grant from funding agencies in the public, commercial, or not-for-profit sectors. 


\begin{abstract}
Aim: Behavioural changes following dementia can have detrimental effects on the quality of life of the individual and family members. In the current service context there is an increasing emphasis on the use of non-pharmacological interventions that aim to both ameliorate behaviour problems and improve quality of life. One relevant clinical framework is Positive Behaviour Support (PBS). This paper introduces the framework, its operationalisation, and some unresolved issues.
\end{abstract}

Methods: Relevant literature and professional experience informs the theoretical and clinical justification for the use of PBS in dementia services, and a case study demonstrates application of the framework.

Results: PBS can be effectively implemented in dementia contexts, but there are significant issues to be resolved regarding resourcing and appropriate goal setting.

Conclusions: Further research is necessary to address skill development among clinicians, a supportive service, and limitations of this framework in the context of a progressive disease process.

Keywords: Positive behaviour support; dementia; challenging behaviour; behavioural and psychological symptoms; quality of life 


\section{Introduction}

Dementia affects 50 million people around the world ${ }^{1}$. It is a degenerative condition that adversely affects cognition, memory and everyday functioning ${ }^{2}$, and often causes changes in aspects of a person's presentation such as agitation, aggression, apathy, disinhibition, wandering, delusions and paranoia ${ }^{3}$. Behaviour changes in dementia are common, with estimates ranging from $61-88 \%$ in a community setting, and up to $90 \%$ in residential care ${ }^{4}$. These changes have detrimental effects on the independence and quality of life of the affected individual and their family, friends, and carers: the latter commonly experience negative impacts on their own health and relationships ${ }^{5}$. Behaviour difficulties in dementia cause greater stress in caregivers than declining cognition and functional impairment ${ }^{6}$, and are a key contributor to earlier transition to residential care ${ }^{7}$. Caregivers may experience feelings of frustration, guilt and hopelessness in the event that family relationships deteriorate as a result of the person's changed behaviours ${ }^{8}$. Family carers may also go through a grieving process as they observe the person's cognitive, functional and social deterioration ${ }^{9}$.

As an individual's functioning declines and behaviour changes occur, family members may find providing informal care at home more difficult, leading to strained relationships with family and friends. Ultimately, with disease progression and behaviour change, transition to residential aged care settings is a common outcome ${ }^{5}$. Although residential care provides a 'bricks and mortar' environment that can help maintain the safety of a person with dementia, and may relieve a family of the ongoing burden of physical care, it is not a method of treatment, and leaves problematic behaviour unaddressed. In the current paper, we discuss treatment approaches, particularly the application of positive behaviour support (PBS) as a framework of support for people with dementia regardless of the stage of dementia or environment in which they live. Specifically, this paper will draw on the relevant literature 
and professional experience in dementia and PBS to provide clear guidance and a theoretical basis for effective use of PBS in the context of dementia.

\section{Intervention approaches}

Currently, interventions to address the behavioural and psychological symptoms of dementia (BPSD) include pharmacology, behaviour intervention, or a combination of both ${ }^{10}$. It is estimated that $70 \%$ of people with dementia are on one or more psychoactive medications ${ }^{11}$. Significantly, pharmacological interventions to manage BPSD are known to have serious potential adverse effects ${ }^{12}$ and deprescribing has become a key aim in the care of people living with dementia ${ }^{13}$.

A significant influence on dementia care approaches has been the use of legislation to direct clinical management practice away from the use of medications that are primarily used for reducing problematic behaviour (termed 'chemical restraint'), and instead direct practice toward improving quality of life ${ }^{14}$. In Australia, the Royal Commission into Aged Care Quality and Safety ${ }^{15}$ investigated the use of restrictive practices (e.g,. physical restraint, chemical restraint) in residential aged care settings. It found an over-reliance on the use of chemical restraint to manage behaviour changes of residents, and also that staff were lacking in knowledge of, and training in, the use of non-pharmacological interventions. The Royal Commission's final report ${ }^{16}$ asserted that the use of restrictive practices in aged care should be prohibited unless "recommended by an independent expert" (p. 221), and should only be used "as a last resort to prevent serious harm after the approved service provider has explored, applied and documented alternative, evidence based strategies to mitigate the risk of harm" (p. 221), which must be stipulated in a behaviour support plan.

As things currently stand, non-pharmacological interventions are increasingly seen as the most appropriate approach in response to behavioural changes, and are recommended as a 
first-line intervention ${ }^{17}$. The rationale for this is straightforward: behaviour support interventions show greater effect in behaviour change than do medications ${ }^{18}$, and many medications have adverse side-effects ${ }^{12}$.

A number of non-pharmacological intervention approaches, with a focus on meaningful community participation and quality of life, have demonstrated clear benefits for people with dementia. These include the Newcastle Model $^{19}$; Tailored Activity Program ${ }^{20}$; Hogewykstyle villages ${ }^{21}$; Environmental Skill-Building Program ${ }^{22}$; Reablement ${ }^{23}$; Alliance Senior Day Services Program and Psychology Practicum ${ }^{24}$; and the Experiential and Wellbeing Model ${ }^{25}$. In practice, these approaches seek to meet needs using individually-tailored approaches, and capitalise on a person's strengths, working with the individual in their own living environment.

PBS is a service response to challenging behaviour ${ }^{26,27}$, which has an increasing evidence base for its application across the lifespan, and with a range of disability types including neurological conditions ${ }^{28-30}$. PBS is presented as the standard framework for promoting best practice behaviour support to people with disability within policy documents and professional guidelines across North America ${ }^{31}$, the United Kingdom ${ }^{32}$, and Australia ${ }^{33}$.

In a preliminary study conducted by Lawson and James ${ }^{34}$, it was suggested that PBS could add value beyond other treatment frameworks. In this study, experts in 'behaviours that challenge' from older adult's services attended a PBS training session and were then asked to evaluate the session and share their views on the application of dementia care services and what PBS could offer to people with dementia. Experts identified many similarities in PBS and existing models used in dementia care (e.g., functional assessments, emphasis on values, and having intervention plans), but suggested that PBS provided a helpful and consistent framework of support, with some potential added benefits. For example, experts reported that 
the principles found in applied behaviour analysis and functional analysis as more sophisticated processes that "go beyond simply describing the behaviour" (p. 18).

Within the present paper we will explain terminology, introduce the PBS framework, and demonstrate its application as a treatment approach for difficult-to-manage behaviour exhibited by individuals with dementia. We also will consider possible constraints to this framework among this clinical group.

\section{Terminology}

In the field of dementia, there are a variety of terms used to refer to challenging behaviours, such as 'behavioural and psychological symptoms of dementia' (BPSD), 'changed behaviours', and 'expressions of unmet need' (e.g., see Dementia Language Guidelines ${ }^{35}$. $\mathrm{BPSD}$, in particular, can be a problematic term because it typically refers both to overt challenging behaviours (e.g., verbal and physical aggression, wandering/elopement) and psychiatric symptoms (e.g., delusions, paranoia). And although they are each encompassed by the one term, it is not the case that each is best served by one treatment/intervention approach - often psychopharmacology. In the current paper we use the term 'challenging behaviour' to refer to overt behaviours such as swearing, hitting, kicking, sexualised comments, and wandering, and refer to them as challenging because they challenge the service system to address them in a way that is effective (decreasing their frequency or severity) and appropriate (consistent with best practice and community expectations). That is, we separate the overt behavioural from the psychiatric symptoms of dementia to help formulation and treatment/intervention. We use the term 'behaviour support intervention' to refer to a personalised plan designed to address behaviour challenges and increase quality of life, using predominantly environmental change (including the physical environment and 
personnel) as a key means of behaviour support, but which most certainly can include treatment with medication.

\section{Positive Behaviour Support}

PBS is a framework for behaviour support intervention that can incorporate a variety of evidence-based approaches, with the intent of increasing a person's quality of life $\mathrm{e}^{26,36}$. Because it involves a clearly-articulated process for assessment and treatment intervention, it can also be applied in a range of situations or contexts in which challenging behaviours occur $^{26}$. Its foundational features are the use of assessment and intervention methods from the field of applied behaviour analysis (ABA), combined with a suite of contemporary service elements (e.g., person-centred practices). Much of the behaviour of interest is considered to be learned and functional, and thereby the controlling variables lay within the individual's learning history, and current environment, and are available for analysis and modification. Hence, these domains are typically the focus of intervention. With such a shift away from primarily (or, only) pharmacological treatment to more individualised and environmentsensitive approaches, an increased agency is provided to personnel charged with support roles, and more self determination provided to the client.

\section{Positive Behaviour Support in Dementia}

The utility of PBS for people with dementia is currently not well understood. There is no literature to guide implementation of the ideologies and framework of PBS for use in this area. As Lawson and James ${ }^{34}$ report, there has been some scepticism and caution regarding the application of PBS for people within a dementia care setting because PBS is associated with learning disabilities and focus on concepts such as teaching new functionally equivalent skills (which may not be fruitful in the context of declining abilities). The progressive decline in cognitive ability of the client, and issues such as length of lifespan, need to be thoughtfully 
navigated as intervention approaches are espoused. We consider this in more detail later in the paper as a potential constraint of the PBS framework.

The key components of PBS are described in the remainder of the paper. Figure 1

summarises these components, and a detailed case scenario can be found in the Appendix to convey the implementation of features of PBS in a dementia context.

Figure 1. PBS Process

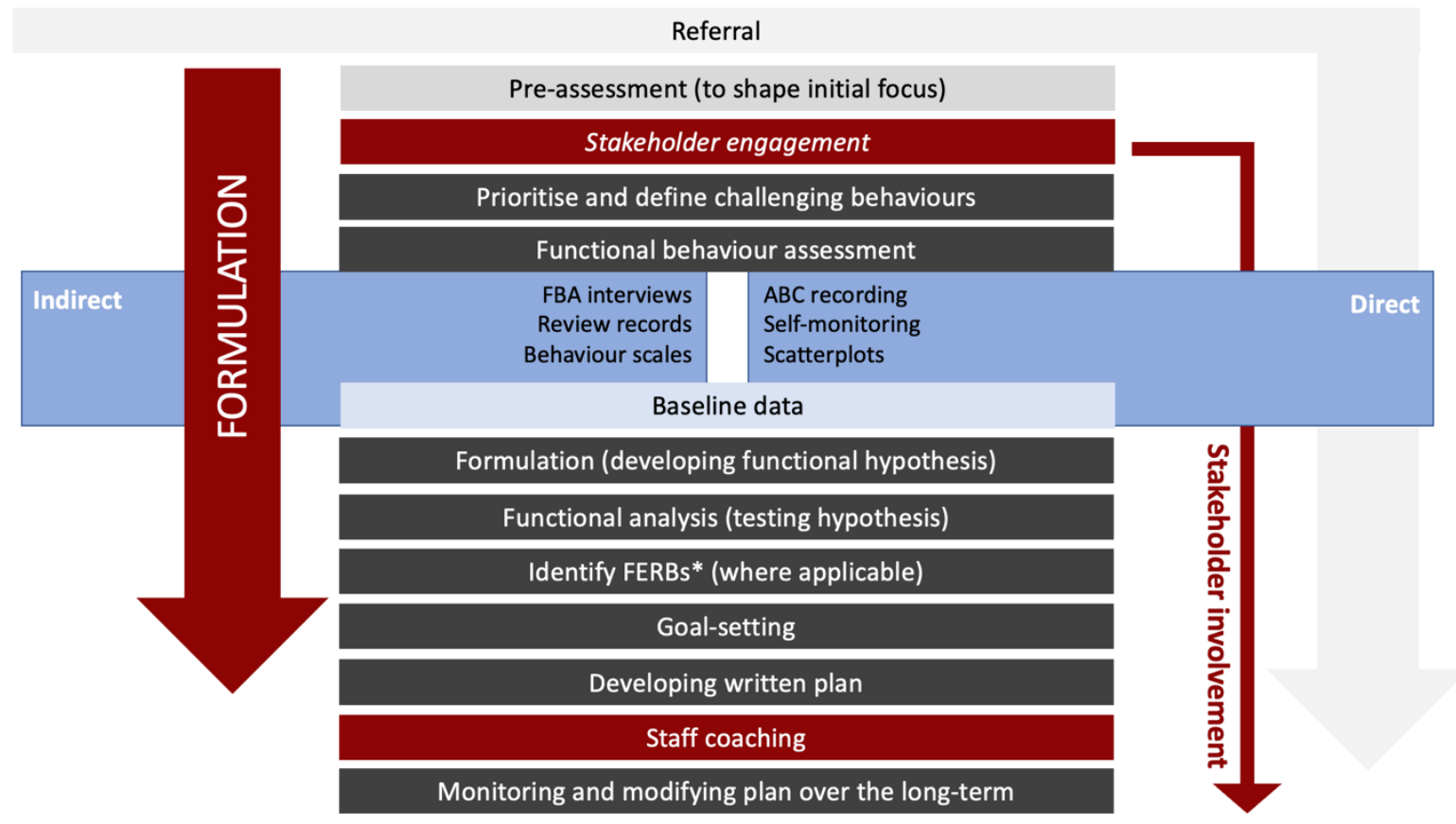

\section{Referral and pre-assessment}

A request for behaviour support could come to a behaviour support practitioner from any one of a variety of sources, such as staff at supported accommodation, general practitioners, allied health or other practitioners, or family members. Pre-assessment then involves collating and evaluating currently-existing information including assessments completed (e.g., neuropsychological assessment report) and details of interventions previously trialled (e.g., 
pharmacology trial and associated behaviour records). Historical and current reports can provide critical medical and functional information, and also important insights into a person's lifestyle preferences (e.g., jazz music), key stakeholders (e.g., engaged family members), and lessons learned from previous interventions (e.g., music therapy leading to engagement and participation). This helps to shape the initial focus of assessment $\operatorname{processes}^{37}$.

\section{Stakeholder engagement}

Stakeholders are defined as the important people in a client's life who will likely be involved in the intervention. Family caregivers, for example, are typically emotionally invested in the support of the client and are likely to play a central role in an ongoing support team ${ }^{38}$. General practitioners are often the most frequently involved medical specialist. Disability support staff are important stakeholders because they often spend more direct hours with the client than anyone else. It is the lived-expertise of family members and support staff with a client that can provide a valuable resource to behaviour support practitioners (e.g., see Fisher et al. $\left.{ }^{39}\right)$. Figure 1 indicates that stakeholder engagement continues through the life of the PBS process.

There is an acknowledgement that stakeholders have 'skin in the game'; they are invested in the clinical care and will have a role - sometimes helpful, sometimes detrimental to positive behaviour change. There is a bi-directional relationship: they can affect the progress of the case, and it can affect them. Hence, a behaviour support practitioner needs to determine a stakeholder's role, their level of engagement, and their ability to implement aspects of an intervention (e.g., collecting behaviour data; using a behaviour change technique). This influences the kind of training delivered to stakeholders, such as behavioural techniques, communication technologies, or the rationale of the PBS model. LeBlanc ${ }^{24}$ noted that shifting 
nursing and clinical staff attitudes away from symptom-based approaches is one of the key indicators for success in a prevention-based model in dementia (such as PBS).

\section{Prioritise and define challenging behaviours}

Because clients with behaviour support needs typically present with more than one challenging behaviour ${ }^{40}$, a determination needs to be made about which behaviours should be targeted for change, and then prioritised. For example, a client may wander many hours a day, potentially abscond/elope, verbally insult staff, physically strike them, destroy important documents, or be noncompliant with a health care regimen.

Behaviour support intervention requires a clear definition or operationalisation of overt behaviour. There are tools that can help a practitioner do this (e.g., the Overt Behaviour Scale ${ }^{41)}$. Clearly defining a behaviour guides stakeholders to a common view of what is to be addressed. Then, prioritising behaviours for change involves the considered use of certain heuristics. Safety is a priority: For example, a client who wanders around their home for many hours per day does not necessarily present a challenge due to wandering per se; but if the client is able to coerce visitors to unlock external doors leading to unsafe environments, then it probably does justify intervention based on risk. Other behaviours may compromise a person's quality of life (e.g., verbal abuse may lead to exclusion from social opportunities). and thereby justify intervention. Prioritising is discussed in detail by Cooper et al. ${ }^{42}$.

\section{Functional behaviour assessment}

Operant behaviour is that which 'operates' on the environment to generate consequences. For example, extending an open palm may be an operant behaviour with a consequence of a friend placing a chocolate in it. Under certain antecedent conditions (e.g., hearing "would you like a chocolate?"), extending an open palm leads to a consequence (receiving a chocolate). 
This chain can be learned and the behaviour will become more frequent if the consequence is reinforcing. Furthermore, there are considered to be four main functions of operant behaviour: escape (e.g., a resident threatens to harm support staff to escape requests to bathe), attention (e.g., a resident phones emergency services regularly, which results in visits from ambulance personnel), tangible items (e.g., pushing another individual to access the television remote) and sensory (e.g., turning down the stereo volume to reduce auditory impact). One of the key tasks in the PBS process is determining the function of challenging behaviour so that this function can be met in a way not requiring the challenging behaviour.

Functional assessment typically involves direct and indirect assessment methods ${ }^{43}$. There is a range of valid functional assessment tools that a practitioner may use, such as the Functional Assessment Interview Form ${ }^{44}$, Functional Analysis Screening Tool ${ }^{45}$, and Brief Functional Assessment Interview Form ${ }^{44}$. Direct assessment of behaviour is typically completed by a behaviour support practitioner, support staff (who are often in situ), or using self-recording. The end result of a functional behaviour assessment will ideally be a clear relationship between a client's motivation (e.g., hunger), the environment (accessible kitchen), the behaviour (rifling through cupboards), the consequence (eating), and the behaviour function (access to sensory gain/enjoyed foods/escape of hunger sensations).

\section{Baseline}

Monitoring behaviour change is a hallmark of applied behaviour analysis ${ }^{42}$, which is a foundation element of PBS. A baseline of target behaviour is essential in order to determine the extent to which a behaviour changes due to intervention. It is important to note that both challenging behaviours (which we want to have decrease) and socially meaningful behaviours (desirable to have increase) should be monitored. A behaviour support practitioner requires a range of skills including: determining which specific behavioural 
dimensions to measure (e.g., typography, frequency, intensity, latency), which intervention design can demonstrate that it is treatment intervention, and not a confounding factor, that is responsible for behaviour change, skill in training direct support staff and family members to collect reliable data, and being able to use data to show behaviour change.

\section{Formulation}

As in other clinical fields, behaviour support practitioners devise formulations or conceptualisations to explain target behaviour using the available information ${ }^{46}$.

Diagnostic and other neuropsychological assessments also play an important role in helping practitioners to understand specific aspects of an individual case that can contribute to challenging behaviours (e.g., behavioural variant frontotemporal dementia). In the field of dementia, there is a well-established understanding of progressive cognitive functional impairment and behaviour change ${ }^{47}$, and these need to be factored into behaviour formulations. Each type of dementia has its own behavioural and cognitive profile, which will present and progress differently for each individual ${ }^{23}$. This has implications for the way that behavioural strategies are developed and implemented ${ }^{47}$.

\section{Functional analysis}

Sometimes there are multiple hypotheses regarding the function of a behaviour. For example, absconding may be explained as an escape of an environment a person perceives as aversive (e.g., a residential aged care facility); But it might instead be pursuit of a preferred food (e.g., from the local pizza shop). Functional analysis is the process of systematically manipulating variables to test assumptions about behaviour (hypothesised function) ${ }^{48}$. In this simple example, if a meal (e.g., pizza) was provided to the resident prior to the time when they would typically abscond, and they did indeed abscond, then this is evidence that supports the 
'aversive environment' hypothesis. They had been deliberately sated for food to reduce the motivation to find food and reduce hunger. Further analysis might reveal that it is a particular staff member and their interpersonal style which precipitates the absconding.

\section{Identify Functionally Equivalent Replacement Behaviours}

A functionally equivalent replacement behaviour (FERB) fulfils the same function as a challenging behaviour, but is a more socially acceptable way of doing so. It may not be the end goal for behaviour change, but is often designed to get change more quickly. For example, saying "chocolate" (assuming a chocolate is provided) is functionally equivalent to snatching a chocolate from the owner. Sometimes this alternate behaviour ("chocolate") is already within a person's behavioural repertoire but needs to be selectively reinforced to become more prevalent, and sometimes a new behaviour needs to be taught ("I want chocolate please").

In supporting a person with dementia, the priority in FERBs is to build the capacity of communication partners to identify and interpret meaning from the person's behavioural cues. When the communication partners understand the function of behaviour, they can then prompt/direct the person to more effective, efficient and appropriate ways to communicate their message. It is important to consider the cognitive capacity of the person and focus on skills and behaviours that already exist in their repertoire ${ }^{49}$.

\section{Goal Setting}

Goal setting refers to stating a certain level of engagement or proficiency in a chosen activity. Goals will be related to increased quality of life and (secondly) decreased challenging behaviour. Importantly, goals are also expected to be closely aligned with a client's values. Goals and values are different. Goals are discrete and finite, and can be 'ticked off' a list, 
whereas values are ongoing, and reflect how someone wants to live their life (e.g., I want to be generous and loving to my children). In the practice of PBS, elucidating values is a therapeutic activity that forms part of a fuller clinical assessment.

Longer-term goals are then broken down into specific behavioural objectives (e.g., to drink at least one litre of water each day of the week). In PBS, constructing specific behavioural objectives that are measurable and closely monitored are essential.

\section{Developing a Written Plan}

A behaviour support plan is a written document, often approximately 10-15 pages in length, that introduces a client, their values and aspirations, their skills, and multi-element interventions to increase quality of life and address challenging behaviours. It would include a combination of antecedent-based (preventative) strategies, skill development techniques and consequent-based approaches. The plan will detail environmental modification (e.g., physical and social), and detail how progress will be monitored. Templates for written plans are readily available online, and funders often require practitioners to use a particular template; This remains a bone of contention in practice, because it is often unclear which audience a funder's template is designed to satisfy: direct support staff, family members, clinicians, or insurance personnel. The audience for operationalising a behaviour support plan are family members, direct support staff, and clinicians. To be effective, it needs to be written for them, be largely jargon free, be the result of an iterative process with its audience, and backed up by face-to-face training by the behaviour support practitioner.

\section{Staff Coaching}

Utilising PBS requires stakeholders who are engaged in direct support to understand behaviour/s as the result of a complex interplay between the person and their environment ${ }^{26}$. 
As a treatment approach then, it is not a challenging person that must change, but a behaviour that challenges us to modify elements of the environment that contribute to the behaviour. This is an important shift in treatment approach for many people affected by dementia, particularly if there is a history of primarily medication management; it puts the onus of behaviour change not just on the individual, but on the social, interpersonal and programmatic environments in which they live ${ }^{36}$.

Coaching is necessary for all people who are tasked with a role in the behaviour support plan. This can be time consuming, difficult to organise, and costly. If successful, however, the great gain is an informed, consistent support approach likely to minimise challenging behaviour, and increase client quality of life. At a case-by-case level, effective staff coaching can be an onerous challenge, but this will become easier as a system of care evolves, and more staff become familiar with frameworks such as PBS, values-based service systems, and applied behaviour analysis techniques. LeBlanc ${ }^{24}$ found that it was beneficial having the behaviour support practitioner included as a part of the core team, working closely with those responsible for day-to-day care of the person with dementia.

\section{Monitoring and Modification}

Measuring target behaviours enables an objective evaluation of whether the goals of the intervention are being met and informs changes to a support plan. The data collected must have good integrity. Charts of target behaviour are critical so that behaviour changes coinciding with intervention changes are apparent. Verbal reports of the direct experience of support personnel working with their client also informs changes to the behaviour support plan. Regular contact between the behaviour support practitioner (charged with updating a written plan) and support network members is critical and one efficient way to do this is for the practitioner to work with staff in their routine meetings (e.g., reflective practice sessions). 
An important issue in behaviour support interventions is determining when they conclude. Unfortunately, interventions often end prematurely due to funding cessation rather than good clinical outcomes. Ideally, interventions will move from an active, resource-intensive, phase to a monitoring phase once environmental elements are updated (e.g., modified gardening tools), support personnel are trained, and natural contingencies take effect (e.g., adult children orchestrating a park visit, and enabling a pleasant experience).

\section{Cautions}

\section{PBS practitioners}

If PBS is to become a common intervention approach in dementia care, it raises the question of which practitioners will do this work? In Australia, there are few career paths to becoming a PBS practitioner, and this is an ill-defined title, and not one regulated by the Australian Health Practitioners Regulation Agency (AHPRA), as are psychologists for example. To demonstrate the fledgling state of the industry, the first university-based Graduate Certificate course $^{50}$ with a focus on supporting people within home and community settings across the lifespan, released its first cohort of PBS practitioners in 2020. For those currently invested in dementia support, the current best option for upskilling staff may be to train existing dementia support staff in PBS approaches; workshops and training is readily found online. As time goes on, more graduates will become available to integrate into dementia support systems.

\section{Progressive decline}

An established element of PBS in the disability and school sectors is skill building. In a dementia context, however, where a person is experiencing a progressive decline in function and, ultimately, end-of-life concerns, it may well be contraindicated to pursue skill building 
to attain functional goals. This need not become an elephant in the room, or a cause to abandon the process; Instead, it can give stakeholders pause for thought as stage-appropriate, values-based goals are devised, and the PBS framework is challenged to flex to a dementia context.

A PBS process often invests resources (time, money, energy) at the front-end to create better long-term outcomes for all involved, but this may not provide funders 'bang for buck' in the context of a progressive disease where "long-term" is too brief to enable cost recovery. It may be that other PBS emphases such as valuing the client at the centre of this process, and deliberately including all stakeholders in planning, will lead to constructed approaches very well suited to the client stage-of-life, realistic (not idealistic) approaches, and establish a support approach and support team equipped to modify interventions as disease progression continues.

\section{Conclusion}

This paper described in some detail the PBS framework and process, with specific consideration in the context of dementia. Effective use of PBS with individuals and families affected by dementia carries great potential for improved behaviour and quality of life outcomes. Nevertheless, for many dementia-related services, the adoption of PBS would be a paradigm shift raising important service issues: what are the necessary staff qualifications and training; what are the advantages and disadvantages of using consultant behaviour support practitioners versus service-embedded ones; what are the limitations of a behaviour support framework developed in school and disability settings when applied to aged care settings with real and immediate end-of-life scenarios?

The development of PBS in dementia care provides a rich vein for further research. For example, where in our existing service systems are the professionals most able to adopt a 
PBS approach demanding interpersonal skills, clinical assessment and formulation, applied behaviour analysis, and so forth? Is it that dementia services require behaviour intervention expertise, or do behaviour support practitioners require improved dementia understanding and who directs these service system changes? For individuals and families affected by dementia, to what extent can this framework, which makes them active agents in change, bring a genuine satisfaction with their quality of life? Although the answers to these questions are far from resolved, we argue that introducing the ethos and practices of PBS to dementia care can only be advantageous to those charged with this responsibility.

\section{Declaration of Conflicting Interests}

The authors (AF, TC, CMOC, GK) report no conflicts of interest to disclose.

\section{References}

1. Alzheimer's Disease International. About Dementia. https://www.alz.co.uk/aboutdementia. Published 2020. Accessed.

2. Quinn JF, Dickinson S. Dementia Chichester, England : Wiley-Blackwell; 2014. 
3. Halloran L. Behavioral and Psychological Symptoms of Dementia. Journal for nurse practitioners 2014;10(8):625-626.

4. Brodaty H, MDraper BM, Low L-F. Behavioural and psychological symptoms of dementia: a seven-tiered model of service delivery. The Medical Journal of Australia $2003 ; 178(5)$.

5. Shnall A, Agate A, Grinberg A, Huijbregts M, Nguyen M-Q, Chow TW.

Development of supportive services for frontotemporal dementias through community engagement. International Review of Psychiatry 2013;25(2):246-252.

6. Machnicki G, Allegri RF, Dillon C, Serrano CM, Taragano FE. Cognitive, functional and behavioral factors associated with the burden of caring for geriatric patients with cognitive impairment or depression: evidence from a South American sample. The American Journal of Geriatric Psychiatry 2009;24(4):382-389.

7. de Vugt ME, Stevents F, Aalten P, Lousberg R, Jaspers N, Verhey FRJ. A prospective study of the effects of behavioral symptoms on the institutionalization of patients with dementia. Int Psychogeriatr 2005;17(4):577-589.

8. Hsiao HC, Chao HC, Wang JJ. Features of problematic eating behaviors among community-dwelling older adults with dementia: family caregivers' experience. Geriatr Nurs. 2013;34(5):361-365.

9. Warchol-Biedermann K, Mojs E, Gregersen R, Maibom K, Millán-Calenti JC, Maseda A. What causes grief in dementia caregivers? Archives of Gerontology and Geriatrics 2014;59(2):462-467.

10. Strydom A, Al-Janabi T, Houston M, Ridley J. Best practice in caring for adults with dementia and learning disabilities. 2016;31(6):42-51.

11. Mesquida MM, Casas MT, Siso AF, Munoz IG, Vian H, Monserrat PT. Consensus and evidence-based medication review to optimize and potentially reduce psychotropic drug prescription in institutionalized dementia patients. BMC Geriatr $2019 ; 19(7)$.

12. Schneider LS, Dagerman K, Insel PS. Efficacy and adverse effects of atypical antipsychotics for dementia: meta-analysis of randomized, placebo-controlled trials. The American Journal of Geriatric Psychiatry 2006;14(3):191-210.

13. Bjerre LM. Deprescribing antipsychotics for behavioural and psychological symptoms of dementia and insomnia. Can Fam Physician 2018;64(1):17-27.

14. National Disability Insurance Scheme (Restrictive Practices and Behaviour Support) Rules 2018 (Austl.). 2018. 
15. Royal Commission into Aged Care Quality and Safety. Restrictive Practices in Residential Aged Care in Australia. Background Paper 4.

https://agedcare.royalcommission.gov.au/sites/default/files/2019-12/backgroundpaper-4.pdf Published 2019. Accessed.

16. Royal Commission into Aged Care Quality and Safety. Final Report: Summary 2021.

17. Magierski R, Sobow T, Schwertner E, Religa D. Pharmacotherapy of Behavioral and Psychological Symptoms of Dementia: State of the Art and Future Progress. Frontiers in Pharmacology 2020;11.

18. Ayalon L. Effectiveness of Nonpharmacological Interventions for the Management of Neuropsychiatric Symptoms in Patients With Dementia. Archives of Internal Medicine. 2006;166(20):2182.

19. James I. The Newcastle Model. In:2017:148-167.

20. O'Connor CM, Clemson L, Brodaty H, et al. The tailored activity program (TAP) to address behavioral disturbances in frontotemporal dementia: a feasibility and pilot study. Disability and Rehabilitation 2019;41(3):299-310.

21. Vinick D. Dementia-friendly design: Hogeweyk and beyond. British Journal of General Practice 2019;69(683):300-300.

22. Gitlin LN, Winter L, Dennis MP, Hodgson N, Hauck WW. Targeting and Managing Behavioral Symptoms in Individuals with Dementia: A Randomized Trial of a Nonpharmacological Intervention. Journal of the American Geriatrics Society 2010;58(8):1465-1474.

23. Poulos CJ, Bayer A, Beaupre L, et al. A comprehensive approach to reablement in dementia. Alzheimers Dement (N Y) 2017;3(3):450-458.

24. Leblanc LA. Integrating Behavioral Psychology Services Into Adult Day Programming for Individuals With Dementia. Behavior Modification 2010;34(5):443458.

25. Power GA. Dementia Beyond Disease. Health Professions Press; 2014.

26. Gore NJ, McGill P, Toogood S, et al. Definition and scope for positive behavioural support. International Journal of Positive Behavioural Support 2013;3(2):14-23.

27. LaVigna GW, Willis TJ. The efficacy of positive behaivoural support with the most challenging behaviour: The evidence and its implications. J Intellect Dev Disabil 2012;37(3):185-195.

28. Feeney TJ, Achilich J. Structured flexibility and context-sensitive behavioral support for the chronically cranky. NeuroRehabilitation 2014;34(4):709-723. 
29. Hassiotis A, Robotham D, Canagasabey A, et al. Randomized, Single-Blind, Controlled Trial of a Specialist Behavior Therapy Team for Challenging Behavior in Adults With Intellectual Disabilities. American Journal of Psychiatry 2009;166(11):1278-1285.

30. O’Connor CMC, Mioshi E, Kaizik C, Fisher A, Hornberger M, Piguet O. Positive behaviour support in frontotemporal dementia: A pilot study. Neuropsychological Rehabilitation 2020:1-24.

31. National Association of State Directors of Developmental Disabilities Services. Position on behavioral support. http://www.nasddds.org/upload. Published 2015. Accessed August 9, 2020.

32. Authority HIQ. National Standards for Residential Services for Children and Adults with Disabilities. https:/www.hiqa.ie/sites/default/files/2017-02/StandardsDisabilities-Children-Adults.pdf. Published 2013. Accessed August 5, 2020.

33. NDIS Quality and Safeguarding Commission. Behaviour Support. National Disability Insurance Scheme. https://www.ndiscommission.gov.au/providers/behavioursupport\#: : :text=Under\%20the \%20NDIS $\% 20$ Commission $\% 2$ C $\% 20$ behaviour,who $\% 2$ Orequire\%20specialist $\% 20$ behaviour\%20support. Published 2019. Accessed August $5,2020$.

34. Lawson E, James I. The application of positive behavioural support to dementia: insights from experts in behaviours that challenge. 2019.

35. Dementia Australia. Dementia Language Guidelines 2018.

36. Lavigna GW, Willis TJ. The efficacy of positive behavioural support with the most challenging behaviour: The evidence and its implications. Journal of Intellectual \& Developmental Disability 2012;37(3):185-195.

37. PBS Coalition. Positive Behaviour Support: A Competence Framework. http://pbsacademy.org.uk/wp-content/uploads/2016/11/Positive-Behavioural-SupportCompetence-Framework-May-2015.pdf. Published 2015. Accessed 27th August 2021.

38. Huis In Het Veld JG, Verkaik R, Mistiaen P, Van Meijel B, Francke AL. The effectiveness of interventions in supporting self-management of informal caregivers of people with dementia; a systematic meta review. BMC Geriatrics. 2015;15(1).

39. Fisher A, Bellon M, Lawn S, Lennon S. Brain Injury, Behaviour Support, and Family Involvement: Putting the Pieces Together and Looking Forward. Disabil Rehabil 2018. 
40. Kelly G, Brown S, Todd J, Kremer P. Challenging behaviour profiles of people with acquired brain injury living in community settings. Brain Inj. 2008;22(6):457-470.

41. Kelly G, Todd J, Simpson G, Kremer P, Martin C. The overt behaviour scale (OBS): A tool for measuring challenging behaviours following ABI in community settings. Brain Inj 2006;20(3):307-319.

42. Cooper JO, Heron TE, Heward WL. Applied Behaviour Analysis 3rd ed. Harlow, UK: Pearson Education Inc; 2020.

43. Lucyshyn JM, Dunlap G, Freeman EA. A Historical Perspective on the Evolution of Positive Behavior Support as a Science-Based Discipline. In: Brown F, Anderson JL, De Pry RL, eds. Individual Positive Behaviour Supports: A Standards-Based Guide to Practices in School and Community Settings. Baltimore, Maryland: Paul H. Brooks Publishing Co.; 2015:3-25.

44. O’Neill RE, Albin RW, Storey KS, Horner RH, Sprague JR. Functional Assessment and Program Development for Problem Behaviour 3rd ed. Stamford, CT, USA: Cengage Learning; 2015.

45. Iwata BA, Deleon IG, Roscoe EM. Reliability and validity of the Functional Analysis Screening Tool. Journal of Applied Behavior Analysis. 2013;46(1):271-284.

46. Sturmey P. Behavioral case formulation and intervention: a functional analytic approach. West Sussex, UK: John Wiley \& Sons Ltd; 2008.

47. O'Connor CM, Clemson L, Hornberger M, et al. Longitudinal change in everyday function and behavioral symptoms in frontotemporal dementia. Neurology Clinical practice 2016;6(5):419-428.

48. Wacker DP, Berg WK, Bassingthwaite BJ, et al. Conducting Functional Analyses of Behaviour. In: Brown F, Anderson JL, Pry RLD, eds. Individual Positive Behaviour Supports: A Standards-Based Guide to Practices in School and Community Settings Brookes Publishing; 2015.

49. O’Connor CM, Wesson J, Clemson L. Active and engaged: maintaining leisure activities in dementia. In: Low LF, Laver K, eds. Dementia rehabilitation: Evidencebased interventions and clinical recommendations. London, UK: Academic Press, Elsevier; 2020.

50. Flinders University. Graduate Certificate in Positive Behaviour Support. https://students.flinders.edu.au/my-course/course-rules/postgrad/gcpbs. Published 2020. 


\section{Appendix}

\section{PBS Case example: Ajay}

Ajay is 69 years old and was diagnosed with dementia 3 years ago. He lives with his wife Sarah who is his primary caregiver. Ajay retired from his career as a plumber a year before his diagnosis, after becoming increasingly confused at times and having made a serious error on large job he was working on. Sarah spoke to their GP, explaining that Ajay has become difficult to motivate, and spends his days watching TV or playing Solitaire on his laptop. Sarah is worried about Ajay's lack of daily activity; she has tried to engage him in activities he used to enjoy, such as cooking dinner, but reports feeling frustrated and becoming upset when Ajay swears at her and tells her to go away. On a couple of occasions recently, Ajay has lashed out physically at Sarah when she was attempting to get him involved in an activity. Ajay and Sarah's GP has referred them for behaviour support intervention.

\section{$\underline{\text { Referral and pre-assessment }}$}

Referral documentation indicates that key stakeholders include Ajay and his wife Sarah, and their children. The challenging behaviours are not well articulated, but appear to be significant enough to warrant intervention. Details on any previous neuropsychological assessments, specific diagnosis of dementia, any medications, and the funding source for behaviour support would need to be clarified in order to complete the pre-assessment process.

\section{$\underline{\text { Key stakeholder engagement }}$}

The ongoing pre-assessment revealed that he has a diagnosis of Alzheimer's disease, has a recent Montreal Cognitive Assessment score of 21/30, and has been identified in neuropsychological testing to have impairments in insight, among other cognitive domains. He requires some prompting to maintain his instrumental activities of daily living such as showering and wearing clean clothes. Ajay and Sarah have two adult children who live 
nearby and are supportive, but are busy with their own young families. Sarah is managing the household and caring for Ajay, but is finding this increasingly difficult because he is requiring more prompting and support.

Ajay's challenging behaviours have become clearer. When Sarah is cooking the dinner, Ajay (who used to be the primary cook) will stand next to her and yell in her face, telling her what to do. When he became apathetic, Sarah contacted the local Men's shed to arrange a weekly visit for Ajay. Recently however, when Sarah wakes Ajay on the Men's shed mornings, he swears at her and doesn't get out of bed. Sometimes, this has escalated to Ajay lashing out physically and trying to hit Sarah; recently, Sarah pulled back his bed covers to try and get him out of bed and he slapped her on the face. Sarah feels overwhelmed with managing the house and making sure Ajay has all his basic needs met, much less addressing his quality of life issues. She feels unsure about how she will keep caring for him if these behaviours continue.

Prioritise and define challenging behaviours

The behaviour support practitioner arranged a visit to Ajay and Sarah in their home. In situ visits are a hallmark of collaborative interventions with families. A range of challenging behaviours were discussed during a semi-structured interview using the Overt Behaviour Scale; these included verbal and physical aggression, reduced initiation, and some socially inappropriate behaviour (malodour; wearing soiled clothing). It was determined that the priority behaviours for change were physical aggression (slapping Sarah on the face) and verbal aggression (yelling at Sarah) because they compromised Ajay's likelihood of remaining living in his own home, and because they caused Sarah the most distress. In addition, Ajay was spending large amounts of time each day either in bed or sitting on the couch watching TV. Although not aggressive during these times, there was concern that this was detracting from his quality of life, and warranted intervention. 


\section{$\underline{\text { Functional behaviour assessment }}$}

The behaviour support practitioner trained Sarah to observe and record the target behaviours. and related events presumed to be relevant to maintaining the behaviour. The data showed that Ajay is physically aggressive only in the mornings in the half hour or so after he has been woken up. This behaviour appears to function as an 'escape' because the antecedent events consistently were Sarah telling Ajay to get up, hurry up, and talking about being on time for the Men's Shed. Ajay showed precursor behaviours (verbal complaint, querying what was going on). Once Sarah stopped talking, Ajay ceased aggression.

The other priority behaviour was yelling in Sarah's face. The data showed that this most often happened when Sarah was cooking and gardening, but not other tasks such as cleaning. The consequence that was maintaining yelling was not clear; Sarah would either persist with the task or take time away, and Ajay sometimes tried the task, or left the scene to watch television. It was speculated that yelling had an escape function. That is cooking or gardening signalled things that Ajay used to manage. He may experience internal 'arousal' sensations that he can lessen (escape) by yelling at Sarah to leave 'his' tasks. Alternatively, this behaviour may serve a tangible/sensory function because Ajay gets access to the task only when Sarah leaves. This detailed understanding of these priority behaviours came from a combination of Sarah's record keeping, the functional behaviour assessment, and a detailed history including knowledge of Ajay's previous interests and roles.

\section{Formulation}

A detailed understanding of Ajay's current functional and cognitive abilities and previous interests have played a vital role in devising a formulation for his case. Understanding his personal context and how this relates to the identified priority behaviours is key to effectively 
formulating a plan to address the behaviours using available resources and stakeholders. For Ajay's yelling, a brief provisional formulation may be as follows:

Ajay has a history of engaging in cooking and gardening. Indeed, he considered cooking and gardening as his domestic domains, and was often praised for his skills in these areas. As a result of the dementia process, he no longer initiates these activities. When Sarah does these tasks, Ajay can see and hear this activity, and approaches her and yells in her face. This yelling causes her to cease the activity, and Ajay often then takes over the activity. The behaviour enables him to escape his unpleasant internal sensations, and also provide him access to a preferred task.

\section{Functional Analysis}

It was formulated that Ajay yelled at Sarah due to her completing the activities he used to lead. Alternatively, however, it may be that she now prepared food favoured by her, not Ajay. Or that she is using his expensive chef's knives, which he does not want damaged. The explanation matters because it determines the type of intervention approach, and thereby the successfulness of behaviour change. It may be possible to have one of the children come to prepare a meal (testing the notion that the person matters); or Sarah might prepare a meal not requiring the chef's knives (testing the notion that the equipment matters). In this example vignette, the notion was tested that Ajay was expert and sought inclusion in meal planning. It was found that when Sarah sought Ajay's opinion about meal preparation, he became very engaged in the activity, and the frequency and severity of yelling greatly reduced.

\section{Identifying functionally-equivalent replacement behaviour (FERB)}

In Ajay's case, it might be that he is prompted to state "I do this", when he wants to do a certain task. Sometimes it is necessary to increase the occurrence of FERBs using techniques like differential reinforcement, with a plan that natural consequences eventually take effect. 


\section{Goal setting}

Working with Ajay and Sarah, the PBS practitioner conducted an assessment of their values across different life domains. To Ajay, it was important to 'be a provider', to 'look after my family', and ways in which these values had been expressed were through his employment, preparing food, and maintaining the garden. Goals were designed to maintain his contribution in these areas of life. Ajay was to assist in meal choices for each day of the week. This was accomplished by a Monday discussion about meal planning, and a timetable of which days and meals he would assist with preparation. He was supported to engage in gardening on two days of the week.

\section{Developing a written plan}

A range of supportive approaches set the scene for successful goal accomplishment. Ajay showed reduced initiation, and delayed responses to Sarah's prompts to get out of bed once woken up. Environmental supports were recruited to help initiation (e.g., a disability support worker trained in prompting and other approaches, clear written task analyses for garden tasks, consistency in schedule - Tuesday is always gardening day with an assistant). Other stakeholders were recruited to assist with rising in the mornings. Rather than Sarah rushing Ajay to suit her schedule, a routine was established in which she would wake him, bring coffee to the bedtable and invite him to sit to it (an approximation to getting out from bed). They would discuss the day (orientation), and one of the adult children would visit with the grandchildren, and go out to preferred locations such as the nearby gardens and cafes. This places preferred activities in the morning, when Ajay is most energetic, and they reinforce his getting out from bed. In clinical work, we often find that stakeholders are often prepared to help but don't do so without a clearly articulated role, and an understanding of what goals are a focus and why.

\section{Monitoring and modification}


In Ajay's case, an important measure might be how many times he did engage in a community outing with one of his children, as a proportion of all possible opportunities. Measuring target behaviours enables an objective evaluation of whether the goals of the intervention are being met, justifies funding for Ajay's supports, and informs changes to a support plan. 